\title{
GAIT Recognition using PAL and PAL Entropy with BPNN, SVM and MDA
}

\author{
Lateshwari, Pooja kaplesh \\ Bhara university Shimla hills
}

\begin{abstract}
Gait recognition is one sort of biometric innovation that can be utilized to monitor individuals without their collaboration. Controlled situations for example banks, army bases and even terminals need to have the capacity to rapidly distinguish dangers and give varying levels of access to distinctive client groups. Gait demonstrates a specific way or way of proceeding onward foot and gait recognition is the procedure of distinguishing a person by the way in which they walk. Gait is less unpretentious biometric which offers the likelihood to distinguish individuals at a distance with no connection or co-operation from the subject and this is the property which makes it so engaging. This paper proposed new system for gait recognition. In this method, firstly binary silhouette of a walking person is detected from each frame. Secondly, feature from each frame is extracted using image processing operation. Here step size length, distance between hands and cycle length are talking as key feature. Here all experiments are done on gait database. Different groups of training and testing dataset give different results.
\end{abstract}

Keywords: Gait Recognition, BPNN, Support Vector Machine (SVM) and Multiple Discriminant Analysis (MDA)

\section{INTRODUCTION}

The first important steps towards preventing unauthorized access are user authentication. User authentication is the process of verifying identity. Traditionally secret word were situated as a string which included number or extraordinary characters and were utilized for validation and these undoubtedly broke yet now Biometric verifications are utilized. Biometric is a field of innovation that uses mechanized routines for distinguishing and checking a man. Continuously applications like in banks, airplane terminals, validations and checks are constantly needed. In such kind of utilizations biometric distinguishing proof systems are utilized.

Every individual has distinctive features subsequently biometric means extraordinary feature of a person. Biometric qualities are of two types:

- Physiological: face, fingerprints, iris, palm print, DNA etc. These are related to body

- Behavioral: are voice and gait. They are related to behavior of the person.

As these physiological qualities does not give great results in low determination and need client collaboration thusly recognition utilizing Gait is more appealing.

Gait analysis: Gait analysis is the systematic study of human locomotion, increased by instrumentation for measuring body developments, body mechanics and the movement of the muscles. Gait analysis is utilized to evaluate, plan and treat people with conditions influencing their capacity to walk. Gait based recognition is more suitable in video surveillance applications because of following advantages:

- Recognition using gait do not need any user cooperation.

- The gait of an individual can be captured at a distance.

- Gait recognition does not require images, that have been captured to be of very high quality and provide good results in low resolution

- Gait recognition, aiming to identify the individuals by the way he walk or move. Gait recognition can be used in a number of different scenarios.

Gait Recognition Systems: Steps involved in gait recognition system are:

- Background Subtraction: in this approach moving objects from background in the scene are identified first. Then some of the background subtraction techniques are applied on it. Background subtraction are classified in to two techniques:

- Recursive technique

- Non Recursive technique

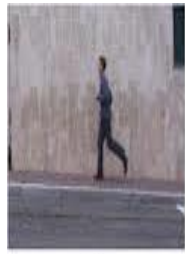

(a)

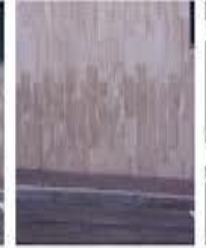

(b)

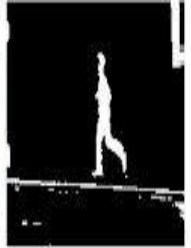

(c)

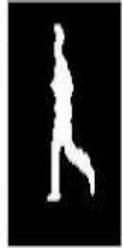

(di)
Figure 1: Background subtraction

- Feature Extraction: feature extraction is an uncommon type of dimensionality decrease. At the point when the input data is so expensive it would be impossible be handled and it is suspected to be famously repetitive (e.g. the same estimation in both feet) then the data information will be changed into a diminished representation set of features (likewise named features vector). Changing the info information into the arrangement of features is called feature extraction. There are some Feature extraction approaches are:

\section{- Model based approaches}

○ Holistic approaches

Holistic methods such as principal component analysis, linear discriminant analysis and independent component analysis used appearance information extracted from entire image. Holistic feature extraction method find feature with reduced 
dimensionality by projecting and original data into basic vectors.

- Recognition: it is the final step of human identification using gait. Here input test videos sequences are compared with sequences in database.

\section{PREVIOUS WORK}

M.Jeevan, Neha Jain, M.Hanmandlu, Girija Chetty (2013) proposed a novel temporal representation of Gait utilizing Pal and Pal Entropy (GPPE) for every cycle of the outlines. The Principal component analysis is connected to each of the features extracted to produce a feature matrix or network. Support Vector Machine (SVM) is utilized for preparing and testing of people by the proposed technique. Broad analysis on the Treadmill dataset and the CASIA datasets A, B, C have been completed to show the viability of the proposed representation of Gait

Alese, B. K., Mogaji, S. A., Adewale, O. S. and Daramola, O.(2012) intends to build up a framework equipped for automatic gait recognition. A individual's gait mark is made utilizing a model based methodology. Transient and spatial metrics extracted from the modular for example length of middle, shin and variation in points of the limb or the abundancy of a persons strolling example can all be utilized to make a "gait signature" of the person which are changed into a self similarity matrix. The utilization of spacio-transient connection strategy to distinguish the subject in ensuing feature groupings.

Jyoti Bharti, M. K. Gupta (2012) Introduced a new gait recognition approach using geometric trademark and fuzzy logic. Proposed a gait framework with the assistance of fuzzy inference system (FIS) for better step acknowledgement rate. This methodology is tried on a database of feature arrangements, relating 17 individuals. The previous methodologies of gait recognition are contrasted with our proposed technique. We get better recognition rate as contrasted with previous strategies. Our method is based on dynamic body parameters. We have taken two segment of human body. The primary segment is hand and second part is feet. The second segment is subdivided into two sections i.e. toe and heel of both right and left leg. Our proposed strategy build the matching accuracy which is lies between 75 to 86 percent.

Lili Liu, Yilong Yin, Wei Qin , Ying Li .(2012) Proposed Gait recognition method based on outermost form . For each gait image arrangement an adaptive silhouette extraction algorithm is firstly utilized to section the frames of the grouping and a progression of post processing is connected to get the standardized silhouette images with less noise. At that point a novel feature extraction system in view of furthest shape is performed. Principal Component Analysis (PCA) is embraced to decrease the dimensionality of the distance signals got from the furthest forms of silhouette images. At that point Multiple Discriminate Analysis (MDA) is utilized to upgrade the distinctness of gait features belonging to distinctive classes. Nearest Neighbor (NN) classifier with respect to class Exemplars (ENN) are utilized to order the last feature vectors created by MDA. So as to check the adequacy and heartiness of our highlight extraction calculation, we additionally utilize two different classifiers - Back propagation Neural Network (BPNN) and Support Vector Machine (SVM) for recognition.
Shalini Agarwal, Shaili Mishra (2012) proposed how to manage the issue of recognizing and following different moving individuals in a static background. Identification of closer object is done by background subtraction. Following different people in complex circumstances is testing. The troubles are handled with fitting information as different models in our methodology. Human movement is decayed into its global motion and limb motion. Our target in this paper is to portion various human objects and track their global movement in complex circumstances where they may move in small gatherings, have entomb impediments, cast shadows on the ground, and reflections may exist.

\section{OBJECTIVES}

Following are the main objectives of this research work:

- To create database of video collected from resources.

- To take input video from user and will cover the distance up to 15 foot.

- Extract all the features of database and input video and match them using model based approach and guarantees quality of result as it considers a bigger number of parameters than beforehand viewed as like separation between hands during movement.

- To analyze Correct Classification Rate by BPNN+MDA + SVM.

\section{METHODOLOGY}

The following steps are the vital steps of proposed work of gait recognition which describes the working of gait recognition system:

Step 1: Select a Live Video for database. It develops a code so that live video is automatically converted into video frames and mat file as database.

Step 2: Select a Live Video for Input. It develops a code so that live input video is automatically converted into video frames. Then it develops a code so that video frames background subtraction using PAL and PAL entropy for input video takes place.

Step 3: It develop a code for noise removal of input video frames. Then It develop a code to extract different features of video frames like height, length of hand, length of leg and distance between left hand and right leg and distance between right hand and left leg.

Step 4: After the feature extraction process it performs recognition process that is the feature of the live database video and the live input video is matched. It develops a code to show the result whether live input video matched with live database video or not.

Step 5: Lastly it develop a code to test recognition performance of our proposed method using BPNN + SVM + MDA which will show better results than previously obtained using other different methods.

\section{RESULTS AND DISCUSSION}

The result of proposed algorithm is highlighted in the following figures:- 


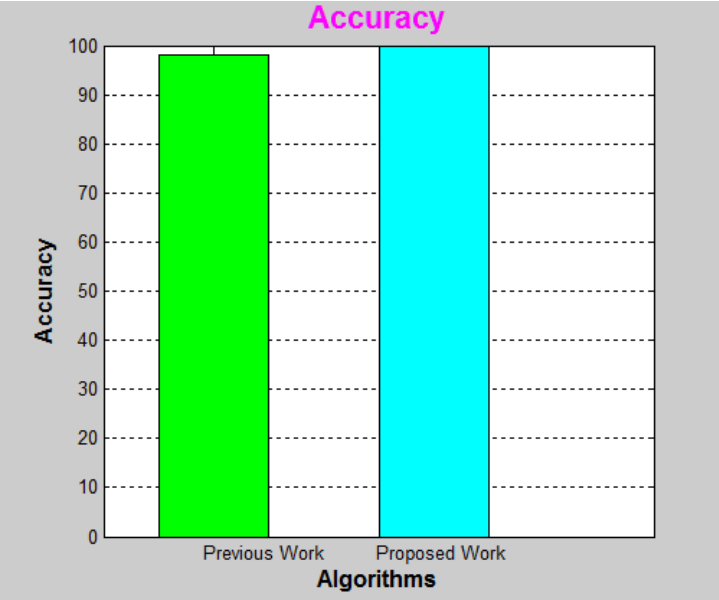

Figure 2: Accuracy of Previous and Proposed work
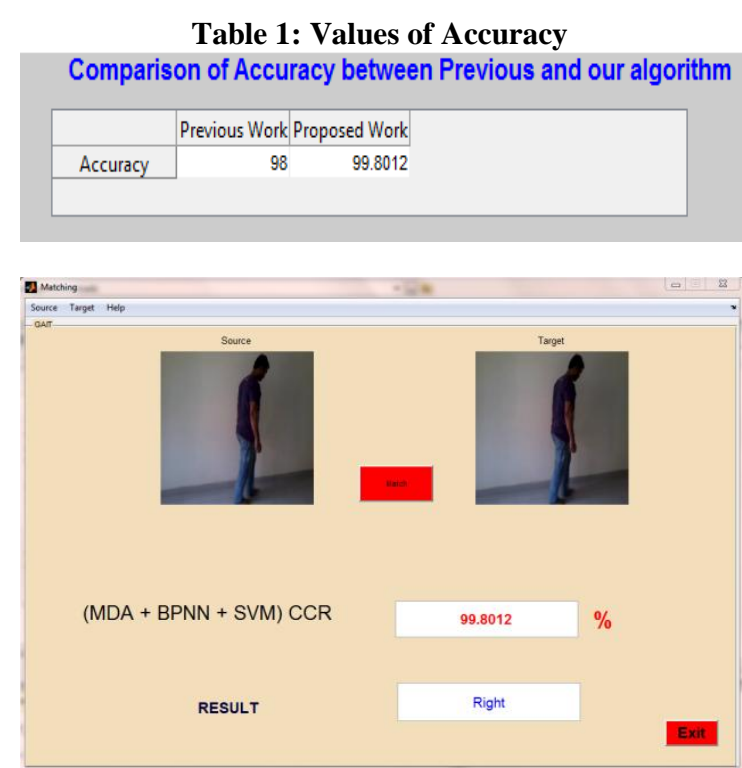

Figure 3: Correct Classification Rate

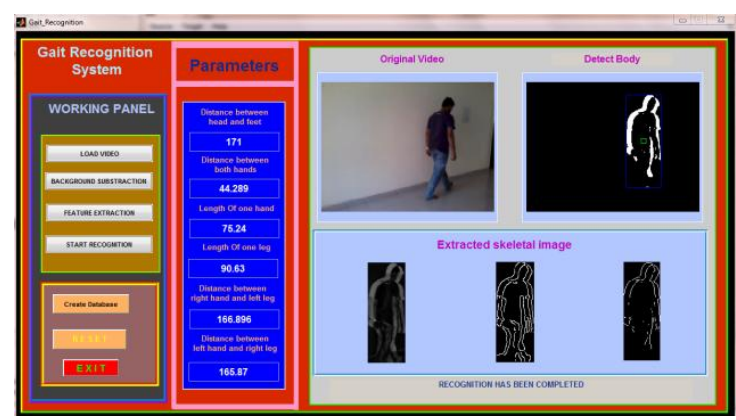

Figure 4: GAIT recognition

\section{CONCLUSION}

The research will involve various areas, which will be highlighted during the course of the study. The study work will make sure that an individual will be recognized using his gait features. And accuracy of gait recognition will be analyzed and compared using different techniques. Some experimental results have shown the effectiveness of proposed system. Shape and boundary information is used for this moving target classification and Width vector of outer contour of binary silhouette and Gait PAL AND PAL ENTROPY coefficients are used for extracting the feature vector then These extracted feature vectors are used to recognizing individual. Surf Feature is used for recognizing persons on the basis of gait and various parameters like distance between hand and distance between legs are calculated .Finally SVM and BPNN+ MDA results are calculated which is far better in comparison to previous research paper.

\section{ACKNOWLEDGEMENT}

Thanks to my Guide and family member who always support, help and guide me during my dissertation. Special thanks to my father who always support my innovative ideas.

\section{REFERENCES}

[1] Jeevan, M., et al. "Indian Institute of Technology Delhi, India." Image Processing (ICIP), 2013 20th IEEE International Conference on. IEEE, 2013.

[2] Alese, B. K., et al. "Design and Implementation of Gait Recognition System." International Journal of Engineering and Technology 2.7 (2012): 1102-1110.

[3] Bharti, Jyoti, and M. K. Gupta. "Gait recognition with geometric characteristic and fuzzy logic." Canadian Journal on Image Processing and Computer Vision 3.1 (2012): 6-11.

[4] Hayder, A., et al. "Person Identification Using Gait." International Journal of Computer and Electrical Engineering 3.4 (2011): 477-482.

[5] Sharma, Sanjeev, et al. "Identification of People Using Gait Biometrics." International journal of machine learning and computing 1.4 (2011): 409-415.

[6] Liu, Lili, et al. "Gait recognition based on outermost contour." International Journal of Computational Intelligence Systems 4.5 (2011): 1090-1099.

[7] N.K Narayanan,V.Kabeer, "Face recognition using nonlinear feature parameter and artificial neural network," International journal of computer intelligence systems, 3(5), 566-574.

[8] Su-Li XY, Qian-jin ZHANG, “ gait recognition using fuzzy principal component analysis", $2^{\text {nd }}$ International Confrence on e-business and information system security, IEEE, 27 may 2010

[9] S.J.McKenna, S.Jabri, Z.Duric, "Tracking group of people," Comput. Vis. Image Understanding, vol.80, no. 1,pp. $42-56$

[10] Davrondzhon Gafurov, Einar Snekkenes and Patrick Bour, "Improves gait recognition performance using cycle matching", International conference on Advanced Information Networking and Applications, Perth, Australia, 20-23 April 2010.

[11] Ali, Hayder, et al. "Gait Recognition using Principal Component Analysis." Proceedings of the 3rd International Conference onMachine Vision. 2010.

[12] Agarwal, Shalini, and Shaili Mishra. "A Study of multiple human tracking for visual surveillance." International Journal 5 (1963). 